\title{
Morcegos (Mammalia, Chiroptera) da Reserva Rio das Pedras, Rio de Janeiro, Sudeste do Brasil
}

\author{
Júlia Lins Luz ${ }^{1,3}$, Luciana de Moraes Costa, ${ }^{1,2}$, \\ Elizabete Captivo Lourenço ${ }^{1}$ \& Carlos Eduardo Lustosa Esbérard ${ }^{1}$ \\ ${ }^{1}$ Laboratório de Diversidade de Morcegos, Instituto de Biologia, \\ Universidade Federal Rural do Rio de Janeiro - UFRRJ, Km 47 da antiga estrada Rio-São Paulo, \\ CP 74507, CEP 23890-000, Seropédica, RJ, Brasil \\ ${ }^{2}$ Programa de Pós-graduação em Ecologia e Evolução, Departamento de Ecologia, Instituto de Biologia, \\ Universidade do Estado do Rio de Janeiro - UERJ \\ ${ }^{3}$ Autor para correspondência: Júlia Lins Luz,e-mail: julialinsluz@yahoo.com.br
}

LUZ, J.L., COSTA, L.M., LOURENÇO, E.C. \& ESBÉRARD, C.E.L. Bats (Mammalia, Chiroptera) from Reserva Rio das Pedras, Rio de Janeiro, Southeastern Brazil. Biota Neotrop. 11(1): http://www.biotaneotropica. org.br/v11n1/en/abstract?article+bn01711012011.

Abstract: A list of bats species from Reserva Rio das Pedras, Municipality of Mangaratiba, was performed using captures with mist nets, from September 1995 to June 2008, totaling 242424 h.net of capture effort. A total of 30 bat species were recorded in 1321 captures and recaptures. The predominant species were Carollia perspicillata and Artibeus lituratus. Six families were registered: Emballonuridae, Noctilionidae, Phyllostomidae, Thyropteridae, Vespertilionidae and Molossidae. Compared with six lists of species available from locations up to $100 \mathrm{~km}$, Reserva Rio das Pedras showed high species richness, diversity and abundance of Phyllostominae, proving the relevance of the site for bats conservation.

Keywords: Atlantic Rainforest, diversity, richness, Phyllostominae.

LUZ, J.L., COSTA, L.M., LOURENÇO, E.C. \& ESBÉRARD, C.E.L. Morcegos (Mammalia, Chiroptera) da Reserva Rio das Pedras, Rio de Janeiro, Sudeste do Brasil. Biota Neotrop. 11(1): http://www.biotaneotropica. org.br/v11n1/pt/abstract?article+bn01711012011.

Resumo: Uma lista das espécies de morcegos da Reserva Rio das Pedras, Município de Mangaratiba, foi elaborada através de capturas com redes de neblina, desde setembro de 1995 até junho de 2008, totalizando 242424 h.rede de esforço de captura. O total de 30 espécies de morcegos foi registrado em 1321 capturas e recapturas. As espécies predominantes foram Carollia perspicillata e Artibeus lituratus. Seis famílias foram registradas: Emballonuridae, Noctilionidae, Phyllostomidae, Thyropteridae, Vespertilionidae e Molossidae. Comparando com seis listas de espécies disponíveis de locais distantes até 100 km, a Reserva Rio das Pedras apresentou alta riqueza, diversidade e abundância de Phyllostominae, comprovando a relevância do local para a conservação dos morcegos.

Palavras-chave: Mata Atlântica, diversidade, riqueza, Phyllostominae. 


\section{Introdução}

No Brasil, a maior parte dos trabalhos com morcegos concentra-se na região sudeste (Bergallo et al. 2003, Esbérard \& Bergallo 2005), em consequência, principalmente, do maior número de universidades e outros centros de pesquisa nesta região (Uieda \& Pedro 1996, Brito et al. 2009). O Estado do Rio de Janeiro é, dentre os estados da região sudeste, o que mais inventários de morcegos possui (Bergallo et al. 2003, Esbérard \& Bergallo 2005). Porém os locais amostrados situam-se principalmente na proximidade da capital e existem várias áreas sem informação ou com um esforço amostral deficiente (Bergallo et al. 2003, Esbérard \& Bergallo 2005, Peracchi \& Nogueira 2010).

Um dos maiores blocos remanescentes de Mata Atlântica situa-se no sudoeste do Estado, com mais de 15.000.000 ha razoavelmente preservados. Este bloco estende-se do litoral a altitudes que ultrapassam 1.000 m, entre os municípios de Mangaratiba e Paraty, onde a maior pressão antrópica reside no desenvolvimento do cultivo da banana, no uso inadequado do solo (Rocha et al. 2003) e na especulação imobiliária (Fidalgo et al. 2009). Apesar da relevância biológica da região, poucos foram os inventários realizados com um número satisfatório de capturas para morcegos (Esbérard \& Bergallo 2005, Esbérard et al. 2006, Dias \& Peracchi 2008, Bolzan et al. 2010).

A subfamília Phyllostominae é conhecida por ser um potencial indicador de áreas menos degradadas, sendo que as áreas com maior riqueza e abundância desta subfamília são consideradas mais preservadas (Fenton et al. 1982, Medellín et al. 2000).
O objetivo deste trabalho foi elaborar uma lista de espécies de morcegos da Reserva Rio das Pedras utilizando dados de levantamentos contínuos e de longo prazo, e compará-la quanto à riqueza e diversidade total de espécies, e a riqueza e porcentagem de capturas de Phyllostominae, com outras seis listas disponíveis para o sudoeste do Estado do Rio de Janeiro.

\section{Material e Métodos}

A Reserva Rio das Pedras situa-se na Praia Grande, km 55 da estrada BR 101, a $8 \mathrm{~km}$ do Município de Mangaratiba e $82 \mathrm{~km}$ do centro da Cidade do Rio de Janeiro (22 $2^{\circ} 59^{\prime} 26,4^{\prime \prime} \mathrm{S}$ e $044^{\circ} 06^{\prime}$ 03,2" W) (Figura 1). Esta reserva possui 1361 ha, que engloba desde a região litorânea até $1150 \mathrm{~m}$ acima do nível do mar, incluindo áreas atualmente abandonadas de cultivo de banana, matas secundárias e primárias. Limita-se ao sul pela Rodovia BR-101, a Leste pela Serra Grande, ao Norte pela Serra Capivari e a Oeste pelo Município de Conceição de Jacareí. A reserva localiza-se na Bacia do Rio Grande e está inserida dentro da Área de Proteção Ambiental de Mangaratiba. Trata-se de uma Reserva Particular do Patrimônio Natural (RPPN), mantida pelo Club Mediterranée.

Foram realizadas 43 noites de captura, uma preliminar em setembro de 1995, 31 noites entre janeiro de 1997 e junho de 1998 , 10 noites complementares entre 2004 e 2006 e uma noite em junho de 2008, compreendendo duas a três noites por mês até 1998 e as demais, realizadas sem critério temporal, geralmente com uma campanha em cada semestre de cada ano. As capturas foram realizadas em todas as fases do ciclo lunar. As redes de neblina foram armadas antes do

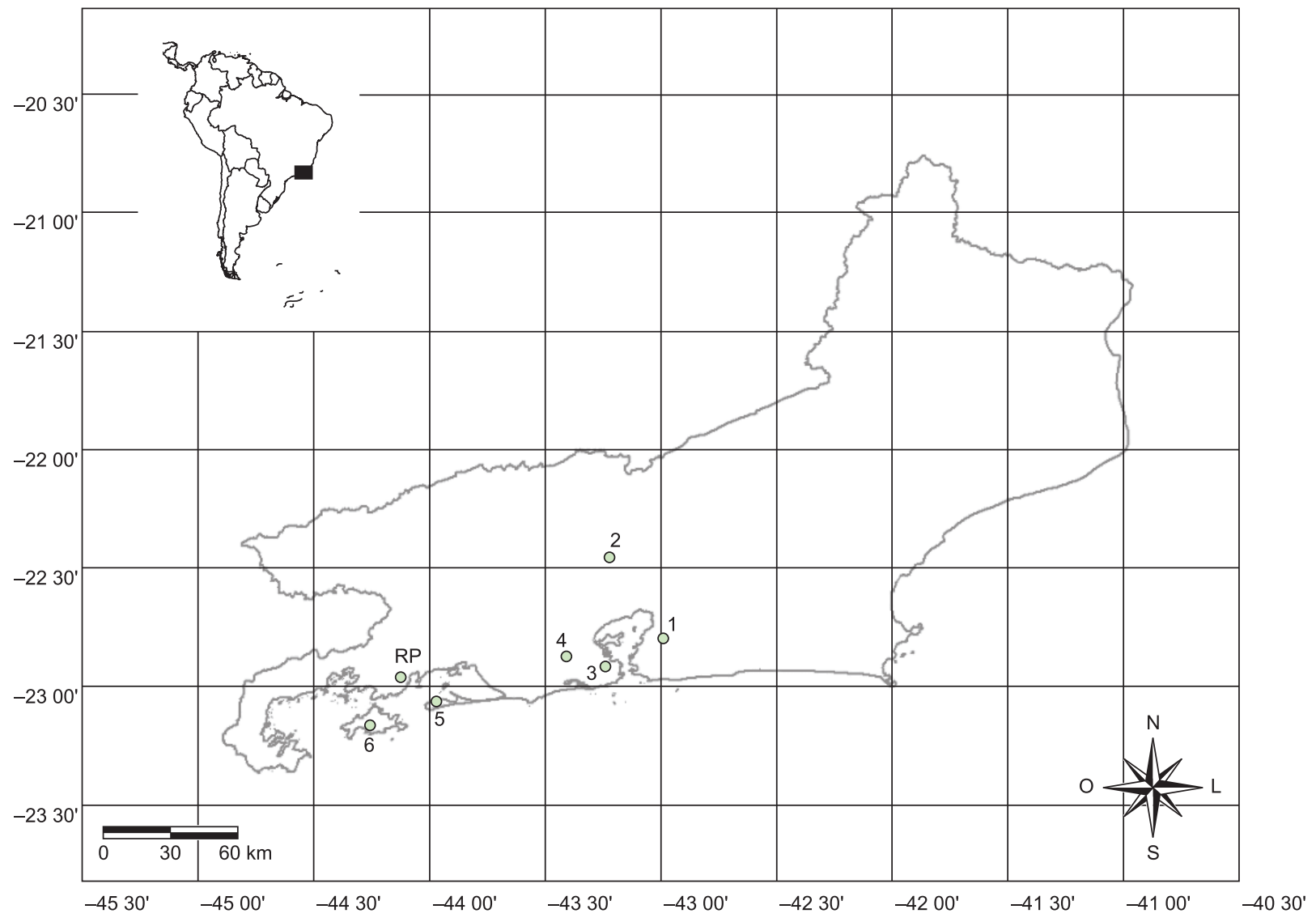

Figura 1. Mapa da América do sul, do Estado do Rio de Janeiro e localização da Reserva Rio das Pedras (RP) e dos demais inventários usados para comparação: 1) Reserva Ecológica Serra da Tiririca; 2) Reserva Biológica do Tinguá; 3) Maciço da Tijuca; 4) Parque Estadual da Pedra Branca; 5) Ilha da Marambaia; e 6) Ilha Grande.

Figure 1. Map of South America, Rio de Janeiro State and location of Reserva Rio das Pedras (RP) and other six sites used for comparison: 1) Reserva Ecológica Serra da Tiririca; 2) Reserva Biológica do Tinguá; 3) Maciço da Tijuca; 4) Parque Estadual da Pedra Branca; 5) Ilha da Marambaia; and 6) Ilha Grande. 
crepúsculo e desarmadas após o amanhecer, totalizando 504 horas e 481 redes. O esforço amostral foi calculado multiplicando-se o número de horas pelo número de redes (h.redes) e a eficiência de captura foi calculada dividindo-se o número de capturas pelo esforço.

As redes foram armadas nas mesmas posições por 28 noites consecutivas até 1998 e após isso foram armadas em outros locais. Três pontos foram amostrados: 1) próximo ao Rio das Borboletas, a cerca de 80 metros da sede da reserva, junto a numerosas rochas, de 1997 a 1998; 2) no Rio Grande, junto a pequeno remanso, distante $410 \mathrm{~m}$ da sede, onde observamos figueira (Ficus citrifolia P. Miller), de 1998 em diante; e 3) sobre o Rio das Borboletas, junto a numerosas figueiras (Ficus enormis (Mart. ex Miq.) Miq. e Ficus gomelleira Kunth) de 1998 em diante.

Os morcegos capturados foram identificados, mensurados e soltos, exceto por um ou dois exemplares de cada espécie que foram sacrificados e preparados, como material testemunho, para depósito na coleção de referência do Laboratório de Diversidade de Morcegos (Processo 1755/89 - IBAMA/SUPES/RJ), Instituto de Biologia, Universidade Federal Rural do Rio de Janeiro (Apêndice 1).

Até 1997, os animais foram marcados com um alicate tatuador, o que permitiu a identificação temporária dos animais (cerca de quatro meses), ou com o uso de colares plásticos providos de cilindros coloridos (Esbérard \& Daemon 1999) a partir de 1998. As recapturas não foram consideradas no cálculo da diversidade. Foi elaborada a curva de acumulação de espécies usando capturas.

Foi utilizado o estimador de riqueza ACE (Abundance-based Coverage Estimator) através do programa SPADE (Chao \& Shen 2010). Esse estimador separa as espécies observadas em dois grupos (raras e abundantes) e utiliza apenas o conjunto das espécies raras para estimar o número de espécies (Chao \& Shen 2010). Foi calculada a porcentagem de espécies capturadas em relação à riqueza estimada para avaliar o quão completo o inventário está. O índice de diversidade utilizado foi o Recíproco de Simpson (1/D) que enfatiza as espécies mais abundantes e é considerado o mais robusto dos índices de diversidade (Magurran 1988).

Comparamos a diversidade, a abundância e a riqueza de Phyllostominae com seis inventários disponíveis para o Estado do Rio de Janeiro (Teixeira \& Peracchi 1996, Dias et al. 2002, Esbérard 2003, Esbérard et al. 2006, Dias \& Peracchi 2008, Lourenço et al. 2010), que estão até $100 \mathrm{~km}$ distantes da Reserva Rio das Pedras, minimizando os possíveis efeitos da latitude (Esbérard et al. 1996, Teixeira \& Peracchi 1996, Dias et al. 2002, Esbérard 2003).

\section{Resultados}

Com um esforço de 242424 h.redes foram obtidas 1228 capturas e 93 recapturas, correspondendo a uma eficiência de 0,005 capturas/h-redes, e 30,70 $\pm 19,76$ morcegos por noite de captura.

Trinta espécies foram amostradas, pertencentes a seis famílias: Emballonuridae (uma espécie), Noctilionidae (uma espécie), Phyllostomidae (23 espécies), Thyropteridae (uma espécie), Vespertilionidae (duas espécies) e Molossidae (duas espécies) (Tabela 1).

O número de espécies capturadas variou de duas a 12 a cada noite, com média de 7,35 $\pm 2,82$ espécies por noite. O número acumulado de espécies atingiu seu máximo na última noite de captura, não apresentando estabilização na curva de acumulação de espécies $\left(\mathrm{y}=5,877 * \operatorname{Ln}(\mathrm{x})-14,30 ; \mathrm{R}^{2}=0,956\right.$ [Figura 2]). A espécie mais abundante foi Carollia perspicillata (Linnaeus, 1758), com 27,56\%, seguida por Artibeus lituratus (Olfers, 1818), com 27,40\% das capturas. Carollia perspicillata foi registrada em 39 noites $(90,70 \%$ das noites de capturas realizadas) e A. lituratus em 41 noites $(95,35 \%$ das noites de capturas realizadas). Carollia perspicillata foi a espécie mais frequentemente capturada em 16 noites $(37,21 \%)$ e A. lituratus em 13 noites $(30,23 \%)$.
O número esperado de espécies pelo estimador ACE (36,9 $\pm 5,4$ espécies) sugere que algumas espécies poderiam ser ainda adicionadas e que a amostragem está de 70,92 a 95,24\% completa. A diversidade de Simpson foi de 1/D = 5,68 \pm 0,27, mostrando-se inferior à média obtida entre as seis localidades utilizadas na comparação (média de $1 / \mathrm{D}=6,90 \pm 1,89$ ), superando apenas duas amostragens, Parque Estadual da Serra da Tiririca e Parque Estadual da Pedra Branca (Tabela 2). A Reserva Rio das Pedras, juntamente com a Ilha Grande, apresenta a maior riqueza de famílias, com seis representadas, enquanto a média nos demais inventários foi de $4 \pm 1,41$ famílias.

A riqueza de Phyllostominae observada na Reserva Rio das Pedras foi alta (seis espécies) e se igualou à encontrada no Parque Estadual da Pedra Branca (Dias et al. 2002), no Maciço da Tijuca (Esbérard 2003) e na Ilha Grande (Esbérard et al. 2006), e a percentagem de captura de Phyllostominae (2,48\% das capturas) foi superada apenas pela registrada no Parque Estadual da Serra da Tiririca (Teixeira \& Peracchi 1996) e pelas amostras da Ilha Grande (Esbérard et al. 2006) e Ilha da Marambaia (Lourenço et al. 2010) (Tabela 2).

\section{Discussão}

A maior parte das listagens disponíveis na bibliografia que empregaram apenas redes de neblina no sub-bosque apresenta menos de 30 espécies (e.g., Peracchi \& Albuquerque 1993, Esbérard et al. 1996, Reis et al. 1996, Taddei \& Pedro 1996, Baptista \& Mello 2001, Dias et al. 2002, Esbérard 2003, Costa \& Peracchi 2005). O número de espécies capturadas na Reserva Rio das Pedras é superado pelo relatado para a Ilha Grande (36 espécies), que dista cerca de $20 \mathrm{~km}$, e que foi inventariada por nove anos, com capturas realizadas dentro de refúgios, com redes no sub-bosque e no dossel (Esbérard et al. 2006), e pelo relatado para a Ilha da Marambaia (34 espécies), distante $15 \mathrm{~km}$, onde cinco diferentes ambientes foram amostrados (Lourenço et al. 2010). Somente duas espécies já capturadas na Reserva Rio das Pedras não foram encontradas na Ilha Grande - Thyroptera tricolor Spix, 1823 e Saccopteryx leptura (Schreber, 1774), e apenas T. tricolor não foi capturada na Ilha da Marambaia.

O número de espécies capturadas no Maciço da Tijuca também foi bastante elevado, no entanto o enorme esforço de captura e número de capturas existente nessa localidade aumenta a probabilidade de captura de espécies que em geral são pouco amostradas. No presente trabalho as capturas se restringiram a áreas próximas da sede da reserva (cerca de $500 \mathrm{~m}$ ) e limitadas a $150 \mathrm{~m}$ de altitude, o que sugere que a riqueza possa estar subamostrada, uma vez que as altitudes mais elevadas não foram amostradas.

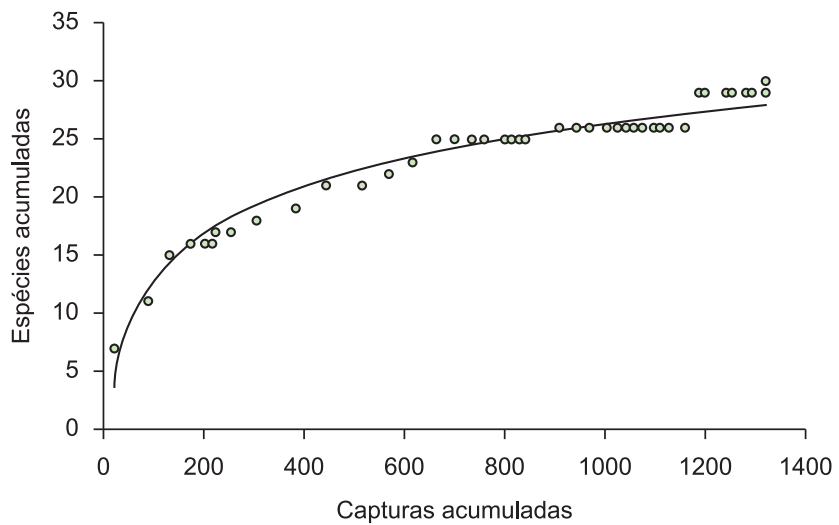

Figura 2. Curva de acumulação de espécies capturadas na Reserva Rio das Pedras, Estado do Rio de Janeiro.

Figure 2. Species accumulation curve for Reserva Rio das Pedras, Rio de Janeiro State. 
Luz, J.L. et al.

Tabela 1. Espécies capturadas na Reserva Rio das Pedras, Estado do Rio de Janeiro, entre 1995 e 2008. As recapturas estão indicadas entre parênteses.

Table 1. Species captured in Reserva Rio das Pedras, Rio de Janeiro, from 1995 to 2008. Recaptures are indicated inside brackets.

\begin{tabular}{|c|c|}
\hline Taxa & Quantidade \\
\hline \multicolumn{2}{|l|}{ Família EMBALLONURIDAE } \\
\hline Saccopteryx leptura (Schreber, 1774) & 1 \\
\hline \multicolumn{2}{|l|}{ Família NOCTILIONIDAE } \\
\hline Noctilio leporinus (Linnaeus, 1758) & 2 \\
\hline \multicolumn{2}{|l|}{ Família PHYLLOSTOMIDAE } \\
\hline \multicolumn{2}{|l|}{ Subfamília Carolliinae } \\
\hline Carollia perspicillata (Linnaeus, 1758) & $364(48)$ \\
\hline \multicolumn{2}{|l|}{ Subfamília Desmodontinae } \\
\hline Desmodus rotundus (É. Geoffroy, 1810) & 34 \\
\hline \multicolumn{2}{|l|}{ Subfamília Glossophaginae } \\
\hline Anoura caudifer (É. Geoffroy, 1818) & $34(1)$ \\
\hline Glossophaga soricina (Pallas, 1766) & 17 \\
\hline Lonchophylla bokermanni Sazima, Vizotto \& Taddei, 1978 & 6 \\
\hline \multicolumn{2}{|l|}{ Subfamília Phyllostominae } \\
\hline Chrotopterus auritus (Peters, 1856) & 3 \\
\hline Lonchorhina aurita Tomes, 1863 & 13 \\
\hline Micronycteris megalotis (Gray, 1842) & 4 \\
\hline Phyllostomus hastatus (Pallas, 1767) & 1 \\
\hline Tonatia bidens (Spix, 1823) & 3 \\
\hline Trachops cirrhosus (Spix, 1823) & $8(1)$ \\
\hline \multicolumn{2}{|l|}{ Subfamília Stenodermatinae } \\
\hline Artibeus fimbriatus Gray, 1838 & $126(7)$ \\
\hline Artibeus jamaicensis Leach, 1821 & $145(11)$ \\
\hline Artibeus lituratus (Olfers, 1818) & $362(14)$ \\
\hline Artibeus obscurus Schinz, 1821 & $103(10)$ \\
\hline Chiroderma doriae Thomas, 1891 & 4 \\
\hline Chiroderma villosum Peters, 1860 & 1 \\
\hline Platyrrhinus lineatus (É. Geoffroy, 1810) & $34(1)$ \\
\hline Platyrrhinus recifinus (Thomas, 1901) & 3 \\
\hline Pygoderma bilabiatum (Wagner, 1843) & 1 \\
\hline Sturnira lilium (É. Geoffroy, 1810) & 18 \\
\hline Sturnira tildae (de la Torre, 1959) & 2 \\
\hline Vampyressa pusilla (Wagner, 1843) & 15 \\
\hline \multicolumn{2}{|l|}{ Família THYROPTERIDAE } \\
\hline Thyroptera tricolor Spix, 1823 & 1 \\
\hline \multicolumn{2}{|l|}{ Família VESPERTILIONIDAE } \\
\hline Myotis nigricans (Schinz, 1821) & 4 \\
\hline Myotis riparius (Handley, 1960) & 10 \\
\hline \multicolumn{2}{|l|}{ Família MOLOSSIDAE } \\
\hline Molossus molossus (Pallas, 1766) & 1 \\
\hline Molossus rufus É. Geoffroy, 1905 & 1 \\
\hline
\end{tabular}

O índice de diversidade e a porcentagem de Phyllostominae na Reserva Rio das Pedras apresentaram valores intermediários, no entanto o número de famílias capturadas e de espécies de Phyllostominae encontradas assemelhou-se às áreas mais ricas. Além da Reserva Rio das Pedras, apenas na Ilha Grande seis famílias foram amostradas, no entanto, os exemplares de Emballonuridae e Furipteridae na Ilha Grande foram capturados dentro de refúgios (Esbérard et al. 2006). A elevada riqueza de Phyllostominae observada pode ser um indicativo da integridade da vegetação, pois estes são considerados os taxa mais sensíveis às modificações da vegetação (e.g., Fenton et al. 1982, Medellín et al. 2000). As maiores riquezas de espécies foram observadas no sudoeste do Estado do Rio de Janeiro, nas localidades amostradas da Ilha Grande, Ilha da Marambaia e Reserva Rio das Pedras (30 a 36 espécies), onde se encontra a maior porção do Estado com remanescentes florestais (Fidalgo et al. 2009). Outros inventários (Peracchi \& Albuquerque 1993, Esbérard et al. 1996, Reis et al. 1996, Taddei \& Pedro 1996, Baptista \& Mello 2001, Dias et al. 2002, Esbérard 2003, Costa \& Peracchi 2005) demonstraram a ocorrência de menos de 30 espécies sugerindo que a conservação de áreas florestadas contribui para a riqueza local. 
Tabela 2. Diversidade (Simpson), riqueza de espécies, capturas, famílias, capturas de Phyllostominae (\%) e riqueza de Phyllostominae para a Reserva Rio das Pedras e outras seis localidades, até $100 \mathrm{~km}$ diatantes, amostradas somente com redes abertas no sub-bosque no Estado do Rio de Janeiro.

Table 2. Diversity (Simpson), species richness, captures, families, captures of Phyllostominae (\%) and Phyllostominae richness at Reserva Rio das Pedras and six other sites, up to $100 \mathrm{~km}$ away, sampled only with mist nets in the understory in Rio de Janeiro State.

\begin{tabular}{ccccccc}
\hline Locais & Diversidade1/D & Espécies & Capturas & Famílias & \% Capturas de Phyllostominae & Riqueza de Phyllostominae \\
\hline RP & 5,68 & 30 & 1.321 & 6 & 2,42 & 6 \\
ST & 4,65 & 20 & 272 & 2 & 5,51 & 5 \\
PB & 4,51 & 24 & 681 & 3 & 2,06 & 6 \\
MT & 8,38 & 40 & 4.042 & 4 & 1,81 & 6 \\
IG & 7,17 & 36 & 3.158 & 6 & 4,91 & 6 \\
RT & 7,79 & 28 & 655 & 4 & 1,22 & 3 \\
IM & 8,90 & 34 & 1.133 & 5 & 4,50 & 4 \\
\hline
\end{tabular}

RP) Reserva Rio das Pedras (este trabalho); ST) Parque Estadual da Serra da Tiririca (Teixeira \& Peracchi 1996); PB) Parque Estadual da Pedra Branca (Dias et al. 2002); MT) Maciço da Tijuca (Esbérard 2003); IG) Ilha Grande (Esbérard et al. 2006); RT) Reserva Biológica do Tinguá (Dias \& Peracchi 2008); e IM) Ilha da Marambaia (Lourenço et al. 2010).

RP) Reserva Rio das Pedras (this study); ST) Parque Estadual da Serra da Tiririca (Teixeira \& Peracchi 1996); PB) Parque Estadual da Pedra Branca (Dias et al. 2002); MT) Maciço da Tijuca (Esbérard 2003); IG) Ilha Grande (Esbérard et al. 2006); RT) Reserva Biológica do Tinguá (Dias \& Peracchi 2008); and IM) Ilha da Marambaia (Lourenço et al. 2010).

Várias espécies amostradas foram raramente relatadas em inventários no sudeste do Brasil. Dentre estas, T. tricolor foi capturada com rede armada sobre o rio, junto a bananeiras (Esbérard et al. 2007). Esta espécie foi citada apenas por Vieira $(1942$, 1955) para os estados do Rio de Janeiro e São Paulo e por Esbérard (2009) para a Ilha da Gipóia, RJ. Lonchorrhina aurita Tomes, 1863 foi capturada junto à figueira em frutificação, onde provavelmente capturava insetos atraídos pela quantidade de frutos em putrefação. Saccopteryx leptura não pode ser considerada rara (Nogueira et al. 2002), porém é uma espécie ainda pouco capturada no Estado do Rio de Janeiro (Esbérard 2009, Lourenço et al. 2010).

Chrotopterus auritus (Peters, 1856) e Trachops cirrhosus (Spix, 1823) são espécies predominantemente carnívoras (Gardner 1977), mais frequentes em ambientes cavernícolas. Estas duas espécies estiveram ausentes no Maciço da Tijuca, onde as amostragens foram realizadas por mais de 10 anos (Esbérard 2003). Apenas Teixeira \& Peracchi (1996), na Serra da Tiririca, Dias et al. (2002), no Parque Estadual da Pedra Branca e Esbérard et al. (2006), na Ilha Grande, citaram estas duas espécies capturadas na mesma localidade no Estado do Rio de Janeiro.

As demais espécies capturadas na Reserva Rio das Pedras mostram-se presentes em todos os levantamentos realizados na Mata Atlântica do sudeste do Brasil que amostraram número razoável de animais (e.g. Peracchi \& Albuquerque 1993, Esbérard et al. 1996, 2006, Reis et al. 1996, Taddei \& Pedro 1996, Baptista \& Mello 2001, Dias et al. 2002, Esbérard 2003). O litoral sul do Rio de Janeiro, que possui um dos maiores remanescentes de Mata Atlântica do sudeste do Brasil, compreende os inventários mais ricos em relação à quiropterofauna, o que demonstra a importância de grandes áreas preservadas na conservação dos morcegos (e.g. Bolzan et al. 2010).

A riqueza total, a riqueza de Phyllostominae e o número elevado de espécies raramente amostradas indicam que a Reserva Rio das Pedras apresenta uma das mais ricas faunas de morcegos do Estado do Rio de Janeiro. Nesta Unidade de Conservação foi confirmada a ocorrência de aproximadamente $40 \%$ das espécies relatadas para o Estado do Rio de Janeiro (Esbérard \& Bergallo 2005, Peracchi \& Nogueira 2010) e cerca de $1 / 3$ das espécies reconhecidas para a Mata Atlântica.

Poucos inventários de morcegos realizados no Estado do Rio de Janeiro apresentam mais de 1000 capturas. Os dados apresentados demonstram a importância de levantamentos contínuos e de longa duração para a captura de um maior número de espécies raras ou pouco frequentes em inventários com redes de neblina, e para obter uma riqueza total elevada.

\section{Agradecimentos}

Ao Club Mediterraneé, mantenedora da Reserva Rio das Pedras, pelo apoio e permissão para coleta. M.A.O. Mocelin, A.S. Chagas, E.M. Luz, A.G. Motta, T. Jordão-Nogueira, D. Moraes e A.F.P.D. Fernades ajudaram no trabalho em campo. A. Pol, D. Dias e A.L. Peracchi (UFRRJ) confirmaram a identificação da maior parte dos espécimes testemunho. O criticismo e as contribuições de H.G. Bergallo, M. Zortéa, W. Pedro, L. Geise, R. Cerqueira e A.L. Peracchi a uma das versões deste trabalho foram de grande valia. Este trabalho foi desenvolvido com apoio da Universidade Gama Filho, Fundo de Conservação Ambiental/SMAC (Processo número 14/001.917/97) e Instituto Biomas e realizado sob a licença especial para coleta (processo 1785/89-IBAMA).

\section{Referências Bibliográficas}

BAPTISTA, M. \& MELLO, M.A.R. 2001. Preliminary inventory of the bat species of the Poço das Antas Reserve, RJ. Chiropt. Neotrop. 7(1-2):133-135.

BERGALlO, H.G., ESBÉRARD, C.E.L., MELlO, M.A.R., LINS, V., MANGOLIN, R., MELO, G.G.S. \& BAPTISTA, M. 2003. Bat species richness in Atlantic Forest: what is the minimum sampling effort? Biotropica 35(2):278-288.

BOLZAN, D.P., LOURENÇO, E.C., COSTA, L.M., LUZ, J.L., NOGUEIRA, T.J., DIAS, D., ESBÉRARD, C.E.L. \& PERACCHI, A.L.P. 2010. Morcegos da região da Costa Verde e adjacências, litoral sul do estado do Rio de Janeiro. Chiropt. Neotrop. 16(1):586-595.

BRITO, D., OLIVEIRA, L.C., OPREA, M. \& MELLO, M.A.R. 2009. An overview of Brazilian mammalogy: trends, biases and future directions. Zoologia 26(1):67-73.

CHAO, A. \& SHEN, T.J. 2010. Program SPADE (Species Prediction And Diversity Estimation). Program and user's guide available at http://chao. stat.nthu.edu.tw (último acesso em 29/09/2010).

COSTA, B.N. \& PERACCHI, A.L. 2005. Morcegos da Ilha da Marambaia. In História natural da Marambaia (L.F.T. Menezes, A.L. Peixoto \& D.S.D. Araújo, eds.). Editora da Universidade Federal Rural do Rio de Janeiro, Seropédica, p.169-194. 
DIAS, D., SILVA, S.S.P. \& PERACCHI, A.L. 2002. Quirópteros do Parque Estadual da Pedra Branca, Rio de Janeiro, RJ (Mammalia: Chiroptera). Rev. Brasil. Zool. 19(2):113-140.

DIAS, D. \& PERACCHI, A.L. 2008. Quirópteros da Reserva Biológica do Tinguá, estado do Rio de Janeiro, sudeste do Brasil (Mammalia: Chiroptera). Rev. Brasil. Zool. 25:333-369.

ESBÉRARD, C.E.L. 2003. Diversidade de morcegos em uma área de Mata Atlântica regenerada no sudeste do Brasil (Mammalia: Chiroptera). Rev. Brasil. Zoociências 5(2):189-204.

ESBÉRARD, C.E.L. 2009. Capture sequence and relative abundance of bats during surveys. Zoologia 26(1):103-108.

ESBÉRARD, C.E.L. \& BERGALLO, H.G. 2005. Research on bats in the state of Rio de Janeiro, southeastern Brazil. Mastoz. Neot. 12(2):237-243.

ESBÉRARD, C.E.L., CHAGAS, A.S., BAPTISTA, M. \& LUZ, E.M. 1996. Levantamento de Chiroptera na Reserva Biológica de Araras, Petrópolis, Rio de Janeiro - I - riqueza de espécies. Rev. Cient. C. Pes. Gonzaga Gama Filho 2:65-87.

ESBÉRARD, C.E.L. \& DAEMON, C. 1999. Novo método para marcação de morcegos. Chiropt. Neotrop. 5(1-2):116-117.

ESBÉRARD, C.E.L., JORDÃO-NOGUEIRA, T., LUZ, J.L., MELO, G.G.S., MANGOLIN, R., JUCÁ, N., RAÍCES, D.S.L., ENRICI, M.C. \& BERGALLO, H.G. 2006. Morcegos da Ilha Grande, Angra dos Reis, RJ, Sudeste do Brasil. Rev. Brasil. Zoociências 8(2):151-157.

ESBÉRARD, C.E.L., SOARES-SANTOS, B. \& FARIAH, D. 2007. New Thyroptera tricolor Spix records in the Atlantic Forest, Brazil (Chiroptera, Thyropteridae). Brazil. J. Biol. 67(2):379-380.

FENTON, M.B., ACHARYA, L., AUDET, D., HICKEY, M.B.C., MERRIMAN, C., OBRIST, M.K., SYME, D.M. \& ADKINS, B. 1982. Phyllostomid bats (Chiroptera: Phyllostomidae) as indicators of habitat disruption in the Neotropics. Biotropica 24(3):440-446.

FIDALGO, E.C.C., UZEDA, C.M., BERGALlO, H.G., COSTA, T.C.C. \& ABREU M.B. 2009. Distribuição dos remanescentes vegetais no Estado do Rio de Janeiro. In Estratégias e ações para a conservação da biodiversidade no Estado do Rio de Janeiro (H.G. Bergallo, E.C.C. Fidalgo, C.F.D. Rocha, M.C. Uzeda, M.B. Costa, M.A.S. Alves, M. Van Sluys, M.A. Santos, T.C.C. Costa, A.C.R. Cozzolino, eds.). Instituto Biomas, Rio de Janeiro, p.91-99.

GARDNER, A.L. 1977. Feeding habits. In Biology of bats of the New World family Phyllostomidae. Part II (R.J. Baker, J.K. Jones Jr. \& D.C. Carter, eds.). Special Publications, Museum Texas Tech University, Texas, p.293-350.
LOURENÇO, E.C., COSTA, L.M., SILVA, R.M. \& ESBÉRARD, C.E.L. 2010. Bat diversity of Ilha da Marambaia, southern Rio de Janeiro State, Brazil (Chiroptera, Mammalia). Brazil. J. Biol. 70(3): 511-519.

MAGURRAN, A.E. 1988. Ecological diversity and its measurement. Croom Helm, London.

MEDELLÍN, R.A., EQUIHUA, M. \& AMIN, M.A. 2000. Bat diversity and abundance as indicators of disturbance in neotropical rainforests. Conserv. Biol. 14:1666-1675.

NOGUEIRA, M.R., PERACCHI, A.L. \& POL, A. 2002. Notes on the lesser White-lined bat, Saccopteryx leptura (Schreber) (Chiroptera, Emballonuridae), from southeastern Brazil. Rev. Brasil. Zool. 19(4):1123-1130.

PERACCHI, A.L. \& ALBUQUERQUE, S.T. 1993. Quirópteros do Município de Linhares, Estado do Espírito Santo, Brasil (Mammalia: Chiroptera). Brazil. J. Biol. 53(4):575-581.

PERACCHI, A.L. \& NOGUEIRA, M.R. 2010. Lista anotada dos morcegos do Estado do Rio de Janeiro, sudeste do Brasil. Chiropt. Neotrop. 16(1):508-519.

REIS, N.R., PERACCHI, A.L., MULLER, M.F., BASTOS, E.A. \& SOARES, E.S. 1996. Quirópteros do Parque Estadual do Morro do Diabo, São Paulo, Brasil (Mammalia: Chiroptera). Brazil. J. Biol. 56(1):87-92.

ROCHA, C.F.D., BERGALLO, H.G., ALVES, M.A.S. \& VAN SLUYS, M. 2003. A biodiversidade nos grandes remanescentes florestais do estado do Rio de Janeiro e nas restingas da Mata Atlântica. Editora Rima, São José dos Campos.

TADDEI, V.A. \& PEDRO, W.A. 1996. Morcegos (Chiroptera: Mammalia) do Vale do Ribeira, Estado de São Paulo: diversidade de espécies. In Anais do VIII Seminário Regional de Ecologia (J.E. Santos, ed.). Programa de Pós-Graduação em Ecologia e Recursos Naturais, Universidade Federal de São Carlos, São Carlos, p.911-919.

TEIXEIRA, S.C. \& PERACCHI, A.L. 1996. Morcegos do Parque Estadual da Serra da Tiririca, Rio de Janeiro, Brasil (Mammalia, Chiroptera). Rev. Brasil. Zool. 13:61-66.

UIEDA, W. \& PEDRO, W.A. 1996. Chiroptera in the XXI Brazilian Zoology Congress. Chiropt. Neotrop. 2(1):41-42.

VIEIRA, C.O.C. 1942. Ensaio monográfico sobre os quirópteros do Brasil. Arquivos de Zoologia do Estado de São Paulo 3(8):219-471.

VIEIRA, C.O.C. 1955. Lista remissiva dos Mamíferos do Brasil. Arquivos de Zoologia do Estado de São Paulo 8(11):341-474.

Recebido em 27/11/2010

Versão reformulada recebida em 20/01/2011

Publicado em 28/01/2011 


\section{Apêndice}

Apêndice 1. Espécimes testemunhos depositados na Coleção de Referência do Laboratório de Diversidade de Morcegos (LDM), Instituto de Biologia, Universidade

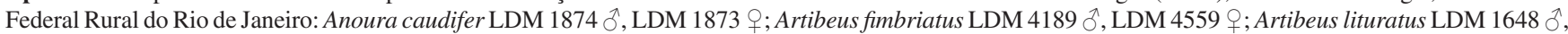

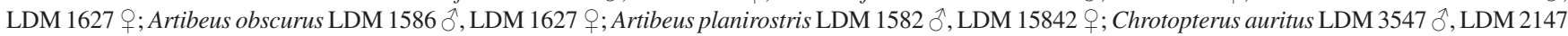

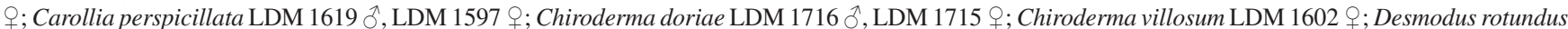

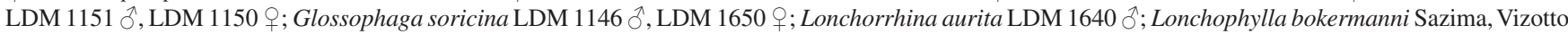

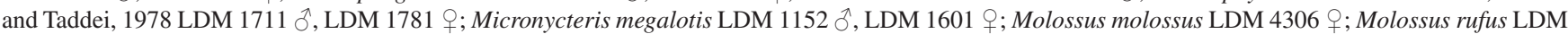

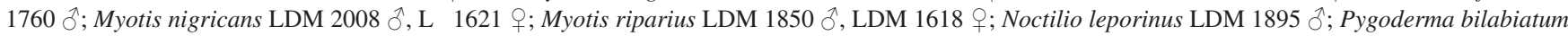
LDM 1853 q; Phyllostomus hastatus LDM 4947 +; Platyrrhinus lineatus LDM 2062 ô, LDM 2061 q; Platyrrhinus recifinus LDM 4367 ô, LDM 4297 of;

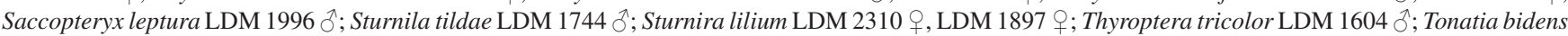

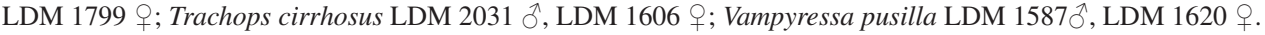

